\title{
Variable modal parameter identification for non-linear mdof systems Part II: Experimental validation and advanced case study
}

\author{
Y.H. Chong and M. Imregun* \\ Imperial College of Science Technology and Medicine, \\ Mechanical Engineering Department, Exhibition \\ Road, London SW7 2BX, UK \\ Tel.: +44 207594 7068; Fax: +44 207584 1560; \\ E-mail:y.h.chong@ic.ac.uk andm.imregun@ic.ac.uk
}

\section{Received 6 June 1999}

Revised 5 January 2000

The purpose of Part II is to provide an experimental validation of the methodology presented in Part I and to consider a representative engineering case, the study of which requires a relatively large numerical model. A beam system with cubic stiffness type non-linearity was used in the experimental study. The non-linear response was measured at three locations and the underlying linear system was obtained via linear modal analysis of low-excitation response data. The nonlinear parameter variations were obtained as a function of the modal amplitude and the response of the system was generated for other force levels. The results were found to agree very well with the corresponding measurements, indicating the success of the non-linear modal analysis methodology, even in the presence of true experimental noise. An advanced numerical case study that included both inherent structural damping and non-linear friction damping, was considered next. The linear finite element model of a high-pressure turbine blade was used in conjunction with three local non-linear friction damper elements. It was shown that the response of the system could be predicted at any force level, provided that that non-linear modal parameters were available at some reference force level. The predicted response levels were compared against those obtained from reference simulations and very good agreement was achieved in all cases.

\footnotetext{
${ }^{*}$ Corresponding author.
}

\section{Experimental verification}

The schematic view of the rig that was designed for the experimental verification of the non-linear modal analysis methodology is shown in Fig. 1. The objective of the experiment was twofold: (i) to validate the harmonic balance program that was used for reference data generation, and (ii) to validate the non-linear modal analysis procedure by comparing directly- measured and regenerated responses for several excitation levels, the latter being obtained from the identified non-linear modal parameters.

The linear part of the rig consists of uniform beam A $\left(420 \times 12 \times 18 \mathrm{~mm}, \mathrm{E}=196 \mathrm{GN} / \mathrm{m}^{2}\right.$ and $\rho=$ $7900 \mathrm{Kg} / \mathrm{m}^{3}$ ) which is clamped at one end. The nonlinear part is formed by two clamped-clamped beams, $\mathrm{B} 1$ and $\mathrm{B} 2$, whose mid points are connected to the free end of the linear beam A. The connection is via a bolt, fastened to a force transducer on one side and to a block mass on the other. With beams B1 and B2 being clamped at both ends, a cubic stiffness type non-linearity is produced by increasing the longitudinal tension under a large vertical displacement. For the purposes of dynamic testing, three accelerometers were placed at $6 \mathrm{~mm}, 82 \mathrm{~mm}$ and $308 \mathrm{~mm}$ from the free end of the linear beam. The experiment consisted of static and dynamic testing of both the linear and non-linear parts.

\subsection{Static tests}

The purpose of the static tests was to confirm the linearity of Beam A and to evaluate the non-linear stiffness coefficient for Beams B1 and B2. During the rig design stage, it was decided that a vibration amplitude larger than $4 \mathrm{~mm}$ would be difficult to achieve in dynamic experiments and the non-linear part was de- 


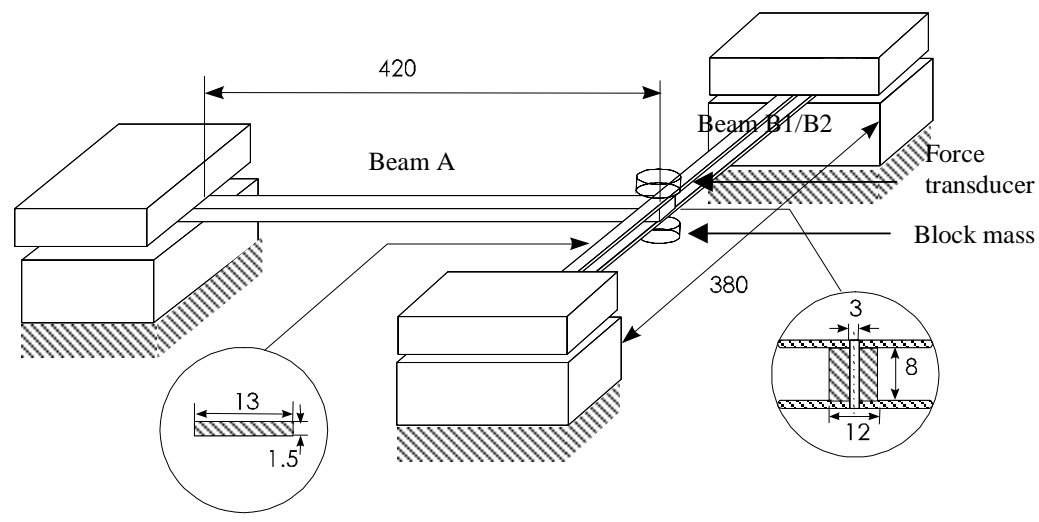

Fig. 1. Experimental setup.

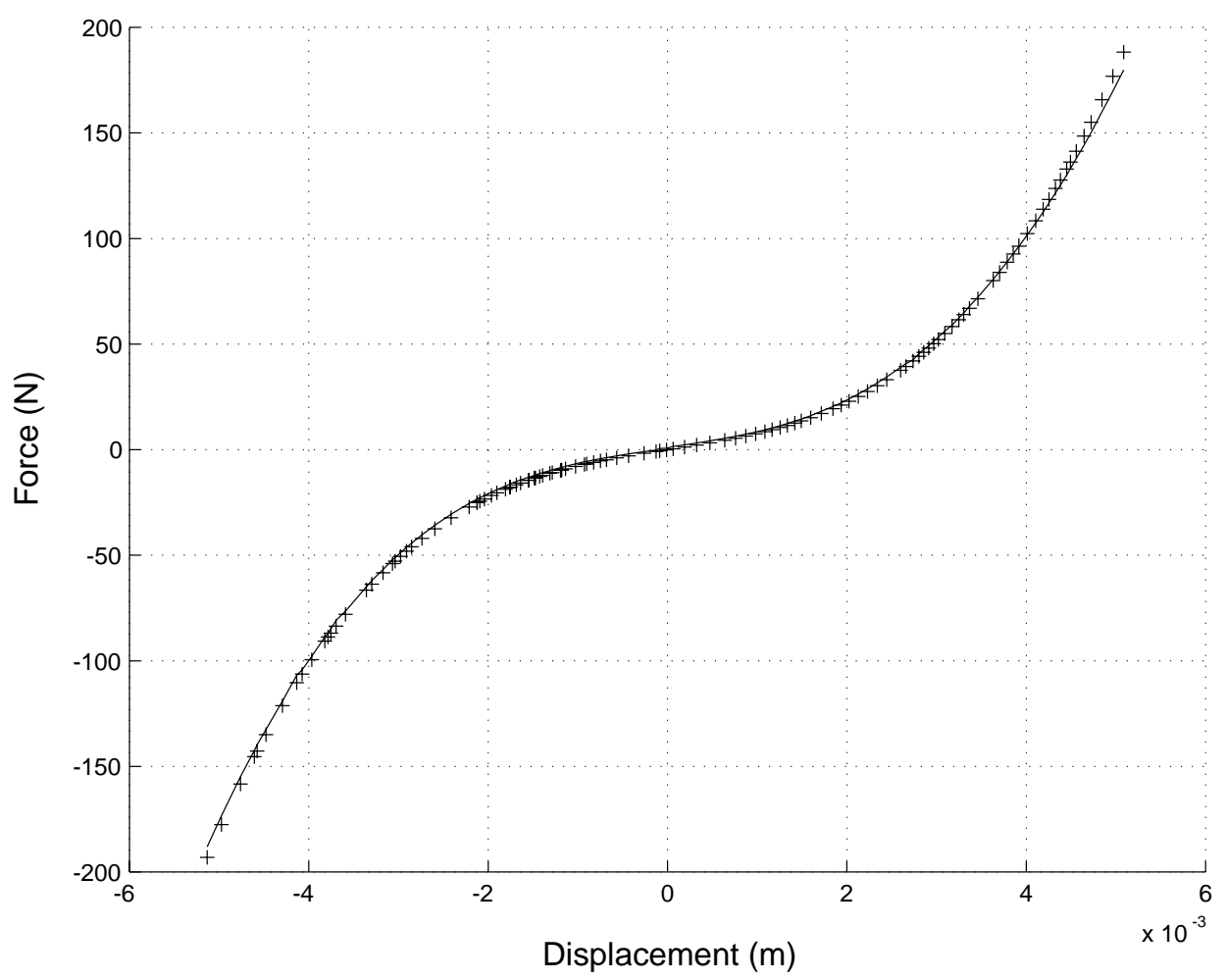

Fig. 2. Displacement-force characteristic for Beams B1/B2.

signed to produce sufficient non-linear force within that range. The tests were carried out using a linear variable displacement transducer (LVDT) and a load cell. The displacement-force characteristic of Beam A was found to be linear while that of Beams B1/B2, shown in Fig. 2, has the expected cubic stiffness characteristic. Its polynomial was estimated as $P=11800 \times 10^{5} x^{3}+6500 x$, where $P$ is the force and $x$ is the displacement. The additional mass due to the non-linear element and sensors was $0.048 \mathrm{Kg}$.

\subsection{Vibration tests}

Two separate dynamic tests were carried out to measure the response of the linear and non-linear parts of the test rig. Here linear test refers to Beam A only while the non-linear test refers to the whole assembly. Interest was confined to the translational degrees of freedom only and sine testing via a suitable frequency response analyser (Beran 402) was used throughout. 


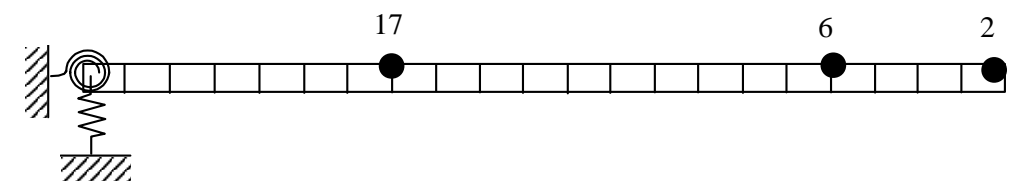

Fig. 3. FE model of Beam A.

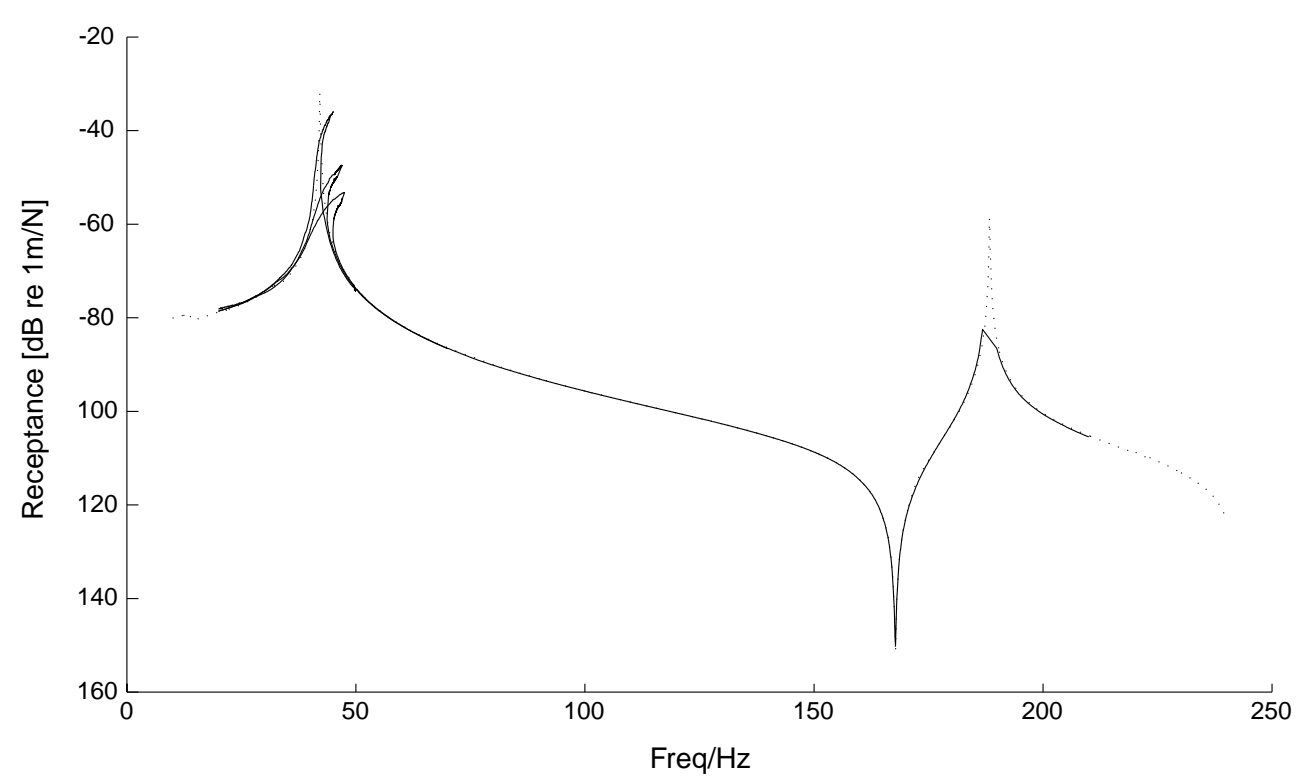

Fig. 4. Non-linear and linearised responses: _ Measured non-linear response, ... Low-excitation (linearised) response.

Beam A was excited for the $0-1500 \mathrm{~Hz}$ range and 4 distinct modes were observed. A finite element (FE) model of Beam A was built in parallel (Fig. 3). At the clamped end of Beam A, a translational spring of 20E6 $\mathrm{N} / \mathrm{m}$ and a rotational spring of $10 \mathrm{KNm} / \mathrm{rad}$ were added to simulate more realistic boundary conditions. The additional mass and stiffness due to the accelerometers, force transducer and shaker's push rod were also incorporated to the model. In addition to the FE modelling, a linear modal analysis was also carried out on the measured FRFs and the response was regenerated from the extracted linear modal parameters. It was found that all three curves, namely the original FRF measurement, the regeneration using linear modal parameters and the FE prediction, were almost identical, a feature that confirms the linearity of Beam A.

Non-linear response measurements on the complete system are much more difficult because of the need to control either the force or the vibration amplitude. An existing data acquisition system, designed to remove the unwanted higher harmonics of the input force signal while controlling its amplitude, was used for this purpose [3]. The non-linear response measurements with feedback control were conducted for the $0-250 \mathrm{~Hz}$ frequency range for force levels of $0.1 \mathrm{~N}, 0.5 \mathrm{~N}$ and $1 \mathrm{~N}$ (Fig. 4). The response was linearised by using the lowest possible excitation and the corresponding curve is also plotted in Fig. 4. It is seen that the non-linearity affects mainly the 1 st mode, probably due to the fact that the response for the 2 nd mode is much lower.

A linear modal analysis of the 1 st and 2 nd modes was carried out and the regenerated response was found to be very close to the linearised response, indicating that a good underlying linear model had been obtained. Next, a non-linear modal analysis was performed on the responses obtained for the $0.1 \mathrm{~N}$ excitation. The extracted non-linear modal parameters are shown in Fig. 5 for the 1 st mode. These are seen to exhibit the expected characteristics of stiffness hardening type non-linearity. While the variation of the natural frequency parameter is well captured by a cubic polynomial, the fit for the $b_{k j}$ parameter is less satisfactory. This is somewhat expected as the $b_{k j}$ parameter is much smaller in magnitude and, as was demonstrated in Part I, more prone to measurement noise.

Once the extracted parameter variations were curvefitted, the non-linear responses were predicted at the 


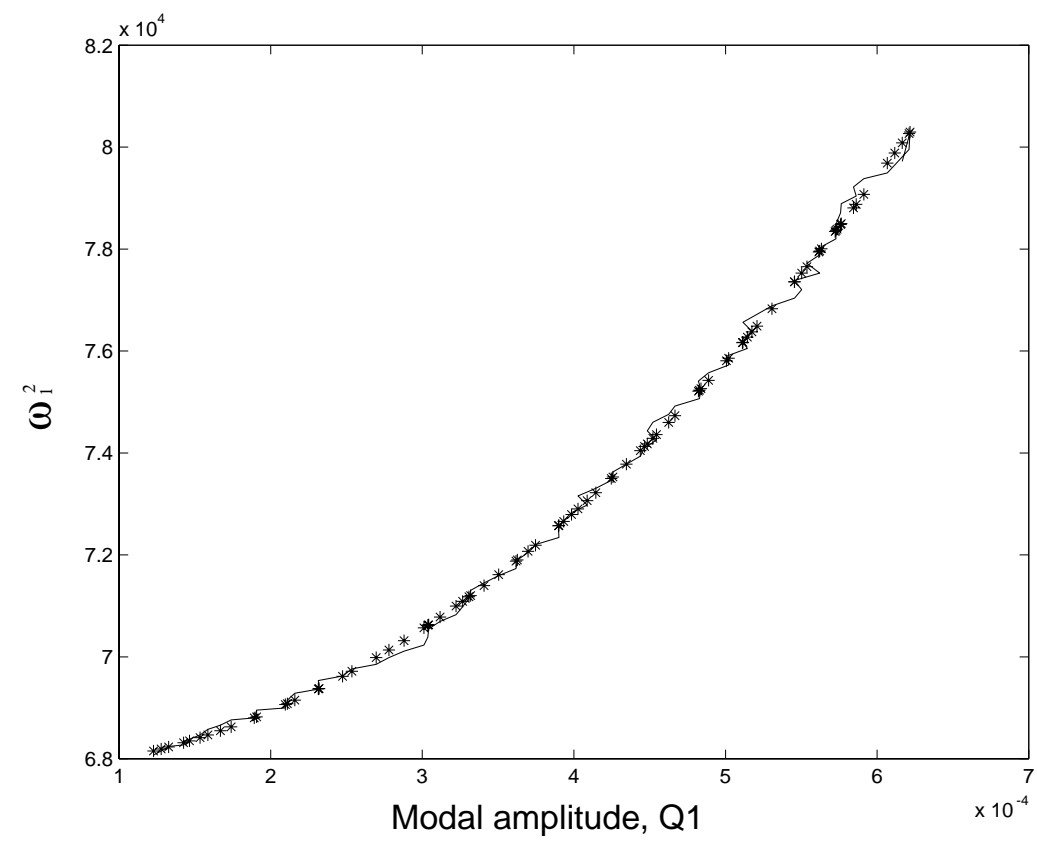

(a) Variation of natural frequency

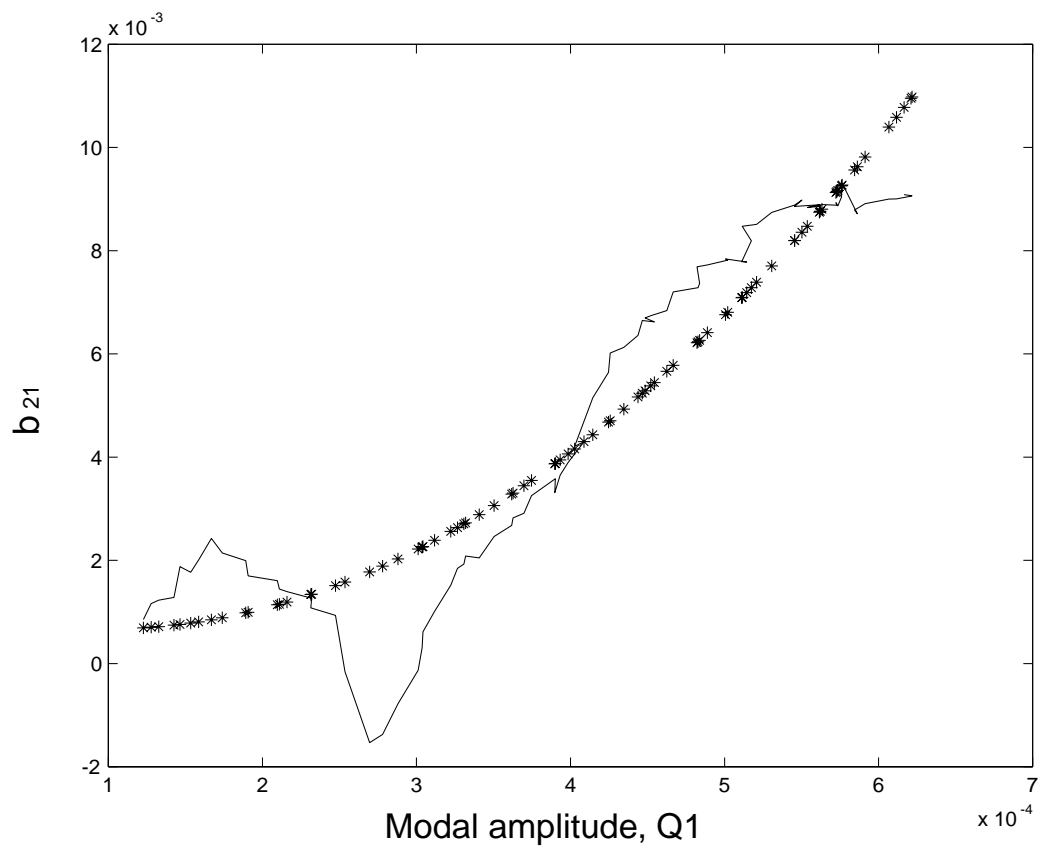

(b) Variation of $b_{21}$

Fig. 5. Non-linear modal parameter variation for the 1st mode: _ Extracted values, ***** Curve-fitted values.

force levels of $0.1,0.5$ and 1 N. Fig. 6 shows the predicted and measured responses, all curves exhibiting a well-known characteristic of non-linear systems, namely multiple states around resonances. These states con- 


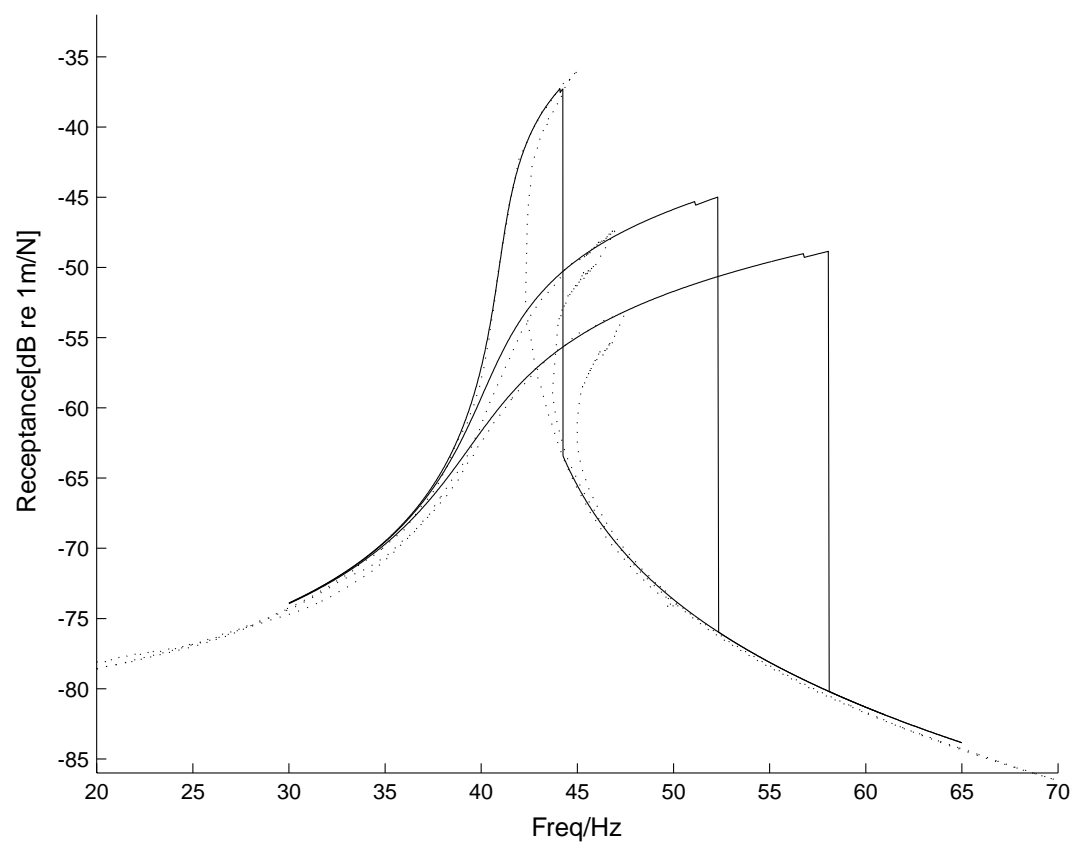

(a) Increasing frequency sweep for numerical prediction

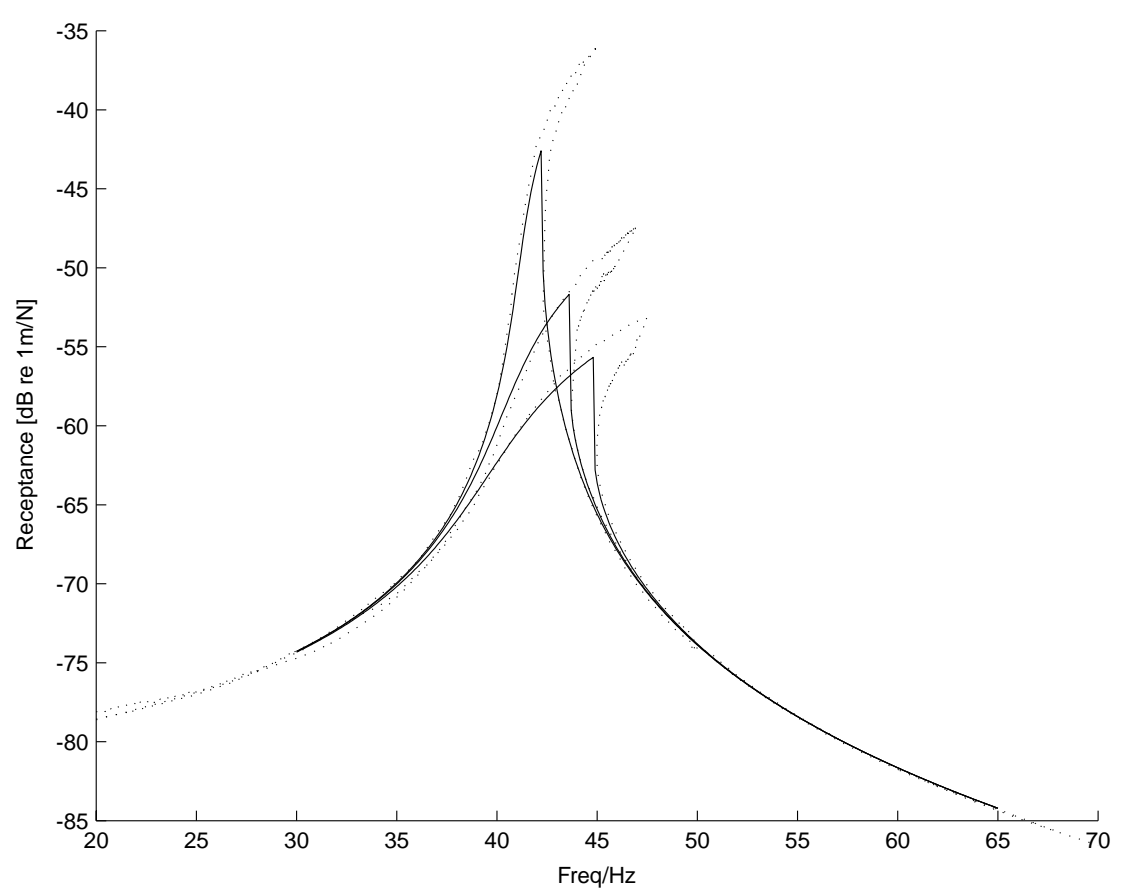

(b) Decreasing frequency sweep for numerical prediction

Fig. 6. Predictions for the 1st mode: _- Predicted response, ... Measured response.

sist of high and low responses on either side of the resonance and of an unstable region around it. In standard measurements, the unstable region cannot be obtained because the structure cannot be made to vibrate in this 


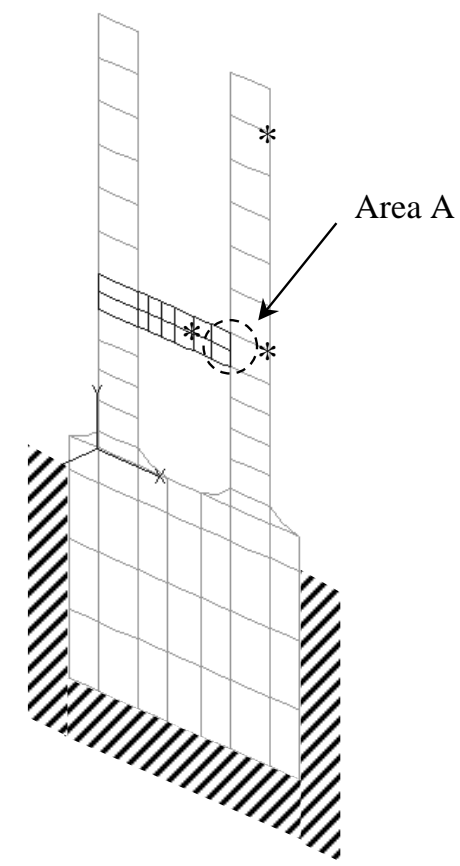

Fig. 7. FE Model of blade: * Measurement point.

particular state. However, the feedback control used here enabled the beam-shaker system to remain stable and continuous response curves were obtained by approaching the resonance from low and high frequencies in two separate frequency sweeps. Therefore the measured response curves are the same in Figs. 6(a) and (b). On the other hand, either increasing (Fig. 6(a)) or decreasing (Fig. 6(b)) frequency sweeps were used during the numerical predictions. In both cases, the solution at the previous frequency was used as an initial guess at the current frequency. With this approach, the response tends to follow the same state until instability is encountered and there is a jump to the higher (or lower) state at this point. Because of the different use of the increasing and decreasing frequency sweeps, the location of the jump phenomenon is different for the measured and the predicted responses. Although it would have been possible to obtain a better agreement by mixing increasing/decreasing frequency sweeps during the numerical simulations, it was decided to present the results as they were first obtained to highlight such subtle differences. In any case, the results demonstrate that is possible to obtain non-linear parameter variations from measured response data. These variations can be used to predict response levels at other force levels. It is now proposed to study a representative engineering case to illustrate the applicability of the methodology to relatively large numerical models with localised non-linearities.

\section{A practical example: turbine blade}

\subsection{Nature of the problem}

High-pressure turbine blades, which are susceptible to blade passing frequency excitation from the upstream stator rows, usually include small pieces of metal which are designed to dissipate energy via friction. Such devices, the so-called friction dampers, are usually placed at the blade roots or between the platforms of two adjacent blades. The centrifugal force provides the normal load and, the friction forces, generated by the blade motion, reduce the amplitude by dissipating energy in a hysteresis-like loop in each vibration cycle. The phenomenon is non-linear since the friction force is a function of the vibration amplitude. The problem faced by industrial designers is twofold: (i) The friction dampers shift the resonant frequency by up to $15 \%$ and this feature needs to be included into the numerical models for accurate forced response predictions [1]. (ii) The normal load, which is a function of the damper mass, rotational speed and radius of rotation, dictates the efficiency of the damper. Performance and geometric considerations determine the last two parameters. If the damper mass is too small, the friction force will not be large enough to dissipate sufficient energy. On the other hand, if it is too large, the damper will not be able to move, limiting the relative motion across the interface, and hence the energy dissipation. Between these two extremes, there will be an optimum damper mass which will provide maximum damping by maximum possible energy dissipation. The determination of an optimum damper mass requires a non-linear vibration model.

There have been numerous studies on the modelling aspects of friction damping [4-7]. One of the key issues is the formulation a representative force- displacement characteristic and several methods are available. The so-called macro-slip model consists of a linear spring and a dry friction element. Until the force reaches the critical level, the force-displacement relationship remains linear. When the force reaches this critical value, its magnitude remains constant regardless of further increases in the displacement values. A more refined version, the micro- slip model, considers the effect of partial slip at some parts of the interface before the gross slip occurs. The load-displacement relationship for the micro-slip model can be expressed as [2]:

$$
F= \begin{cases}s x-t x^{2} & \text { if } 0<x<\frac{s}{2 t} \\ \frac{s^{2}}{4 t} & x \geqslant-\frac{s}{2 t}\end{cases}
$$




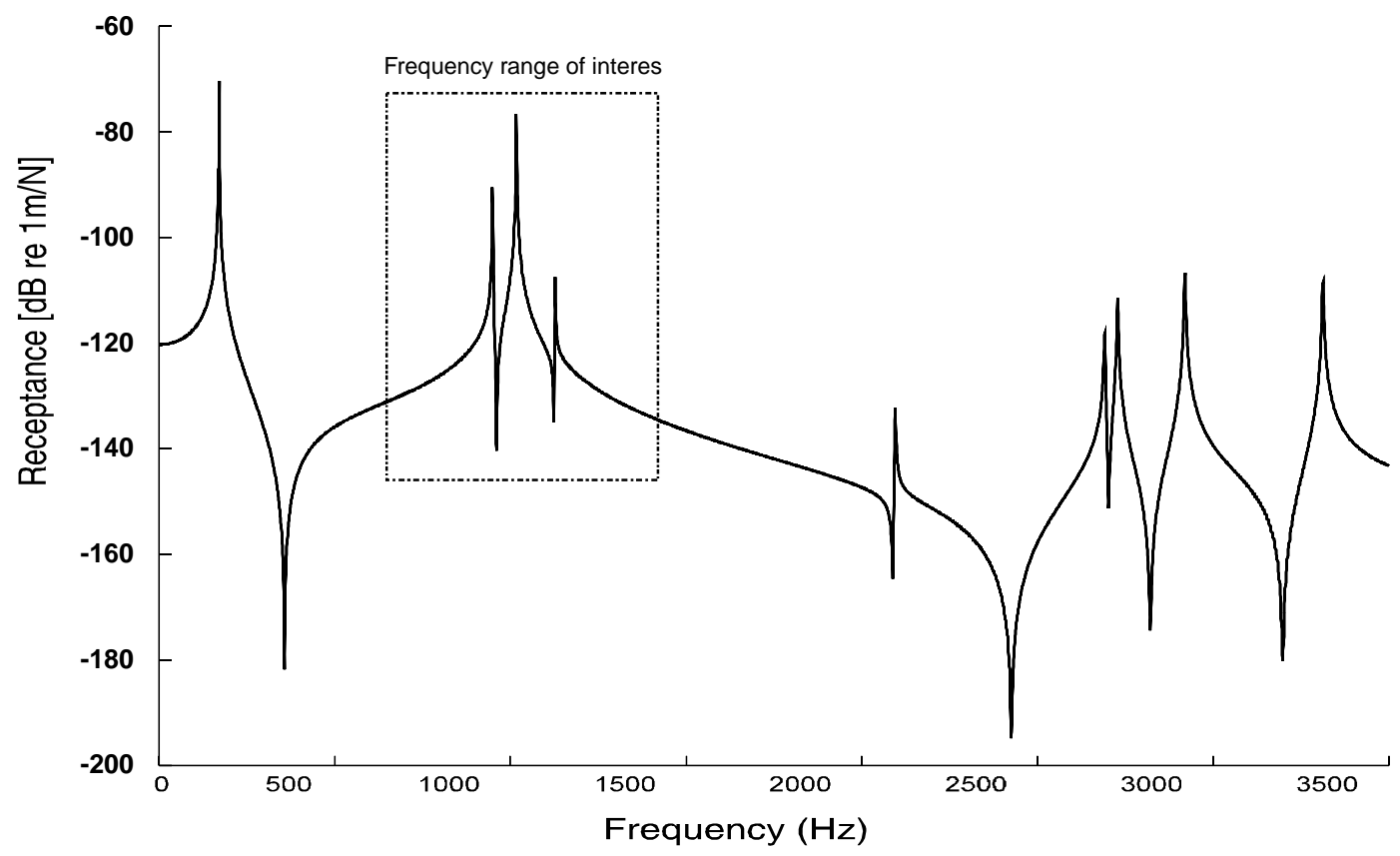

Fig. 8. Frequency response of the turbine blade.

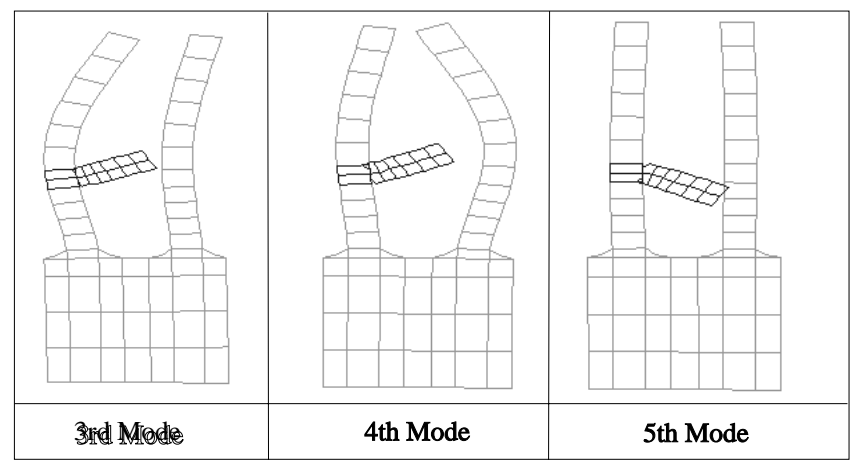

Fig. 9. 3rd, 4th and 5th mode shapes.

where the parameters $\mathrm{s}$ and $\mathrm{t}$ depend on several factors, such as the effective contact area, surface asperity, friction coefficient, etc.

\subsection{Case study}

\subsubsection{Preliminaries}

The non-linear modal analysis technique will now be applied to the simplified blade-damper-blade assembly of Fig. 7. The same case was also studied by $[7,8]$ who predicted the non-linear response under various normal load conditions using a HBM simulation. Their work also included the experimental determination of a force-displacement characteristic which will also be used here. The objective of the current work is to predict the forced response under various excitation conditions from a non-linear modal analysis of (simulated) measurements taken at a single excitation condition.

The linear part of the blade was modelled using 8- and 6-node 2D isoparametric plate elements. The FE model consisted of 300 nodes, each having 2 DOFs. The following material properties were used: Young's Modulus $E=211 G N / m^{2}$, Density $\rho=7800 \mathrm{Kg} / \mathrm{m}^{3}$, Poisson's ratio $v=0.3$. Figure 8 shows the linear response of the blade for the $1-3500 \mathrm{~Hz}$ frequency range. Out of 10 modes, interest was confined to the 3rd, 4th and 5th modes whose shapes are shown in Fig. 9. Three non-linear friction damper el- 


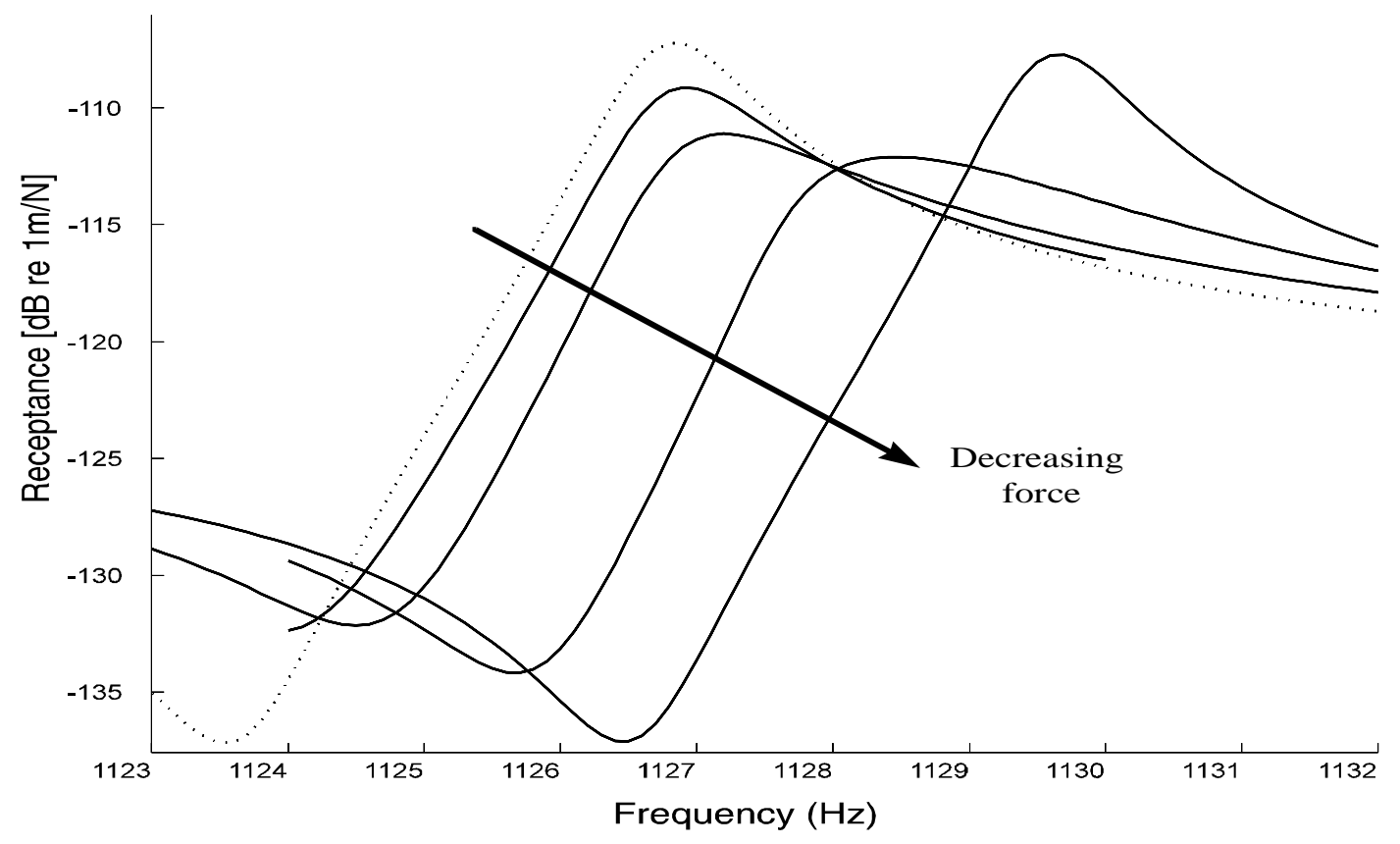

Fig. 10. Blade response at different excitation force levels: . . . Linear response.

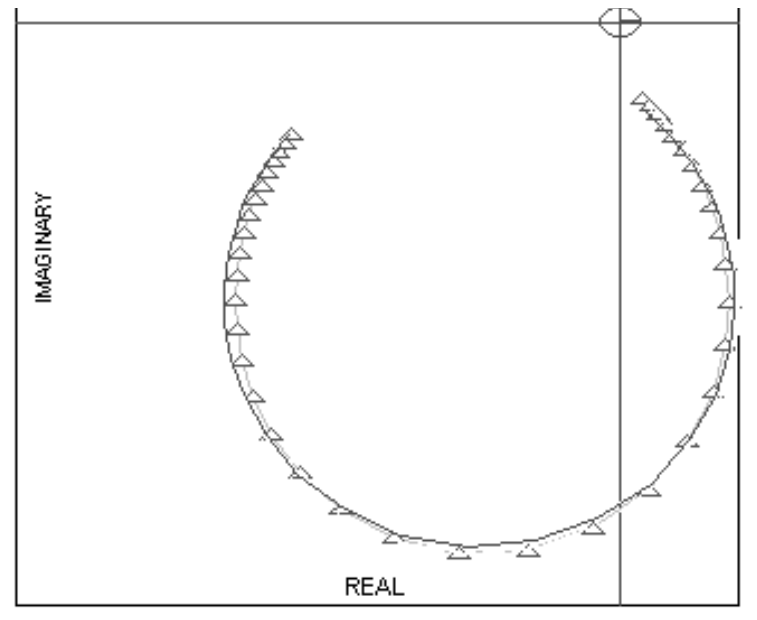

(a) Curve-fit

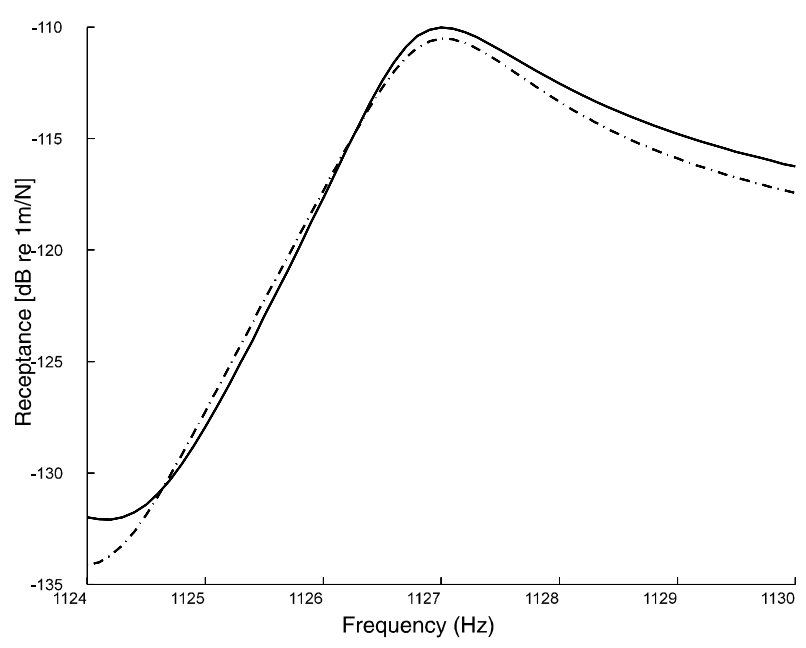

(b) Regeneration from linear model

Fig. 11. Linear modal analysis of non-linear response: _- Original response, ... Regeneration with linear modal parameter.

ements were attached along area A between the blade and the shroud (Fig. 7). The friction damping characteristics used for the simulations were: the contact stiffness $K_{d}=550 \mathrm{~N} / \mathrm{m}$, the critical force level $F=20 \mathrm{~N}$ and the empirical parameter $\beta=0.01$. The parameters were chosen to introduce a noticeable amount of non-linearity into the structure.

\subsubsection{Determination of an underlying linear model}

Before an actual non-linear modal analysis can be conducted, it is necessary to identify the underlying linear model which is not readily available in this case. It was decided to obtain non-linear responses for a number of excitation levels and see if the underlying linear model could be obtained via linear model analysis. Such an approach simulates a typical vibration test 


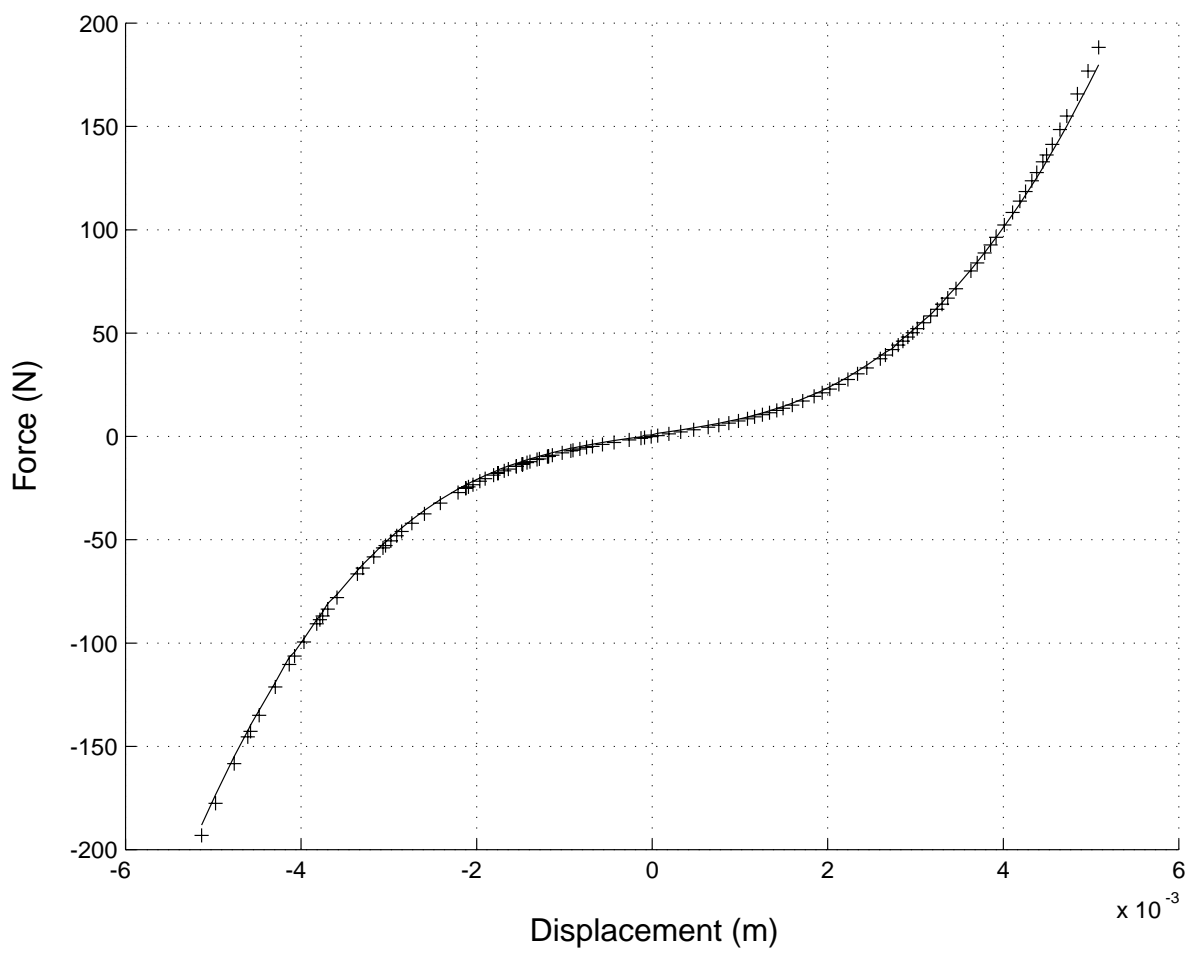

Fig. 12. Linear modal analysis of HP turbine blade with friction damping non-linearity: ... True linear parameters,-- Pseudo linear parameters.

where the response data are acquired for several forcing levels in order to obtain a best experimentally-derived model. The linear eigensolution of the FE model and the friction damper elements were used to compute a column of non- linear responses via HBM simulations, the selected "measurement" points being shown in Fig. 7. Figure 10 shows the blade's response in the vicinity of the 5th mode under force levels of 1000 , 4000,6000 and $9000 \mathrm{~N}$.

To assess the influence of the underlying linear model for non-linear analysis, a series linear modal analyses were performed for the above (simulated) response data. The findings are summarised in Fig. 12 and some aspects are discussed below.

- A linear modal analysis may produce good curvefits but the regenerated response may be significantly different from the original response measurement. Such a situation is shown in Fig. 11 where a linear modal analysis has been conducted on the non-linear responses obtained for a $6000 \mathrm{~N}$ excitation force level. The curve-fit is good for the Nyquist circle but the regenerated response reveals the inadequacy of the linear model.

- For the cases considered, mode shape errors were found to be smallest at low or high excitation levels.
- The non-linearity is provided by the friction damper, whose behaviour is linear not only at low force levels (small amplitude) but also at high force levels (no relative motion because of the damper locking). Therefore, a linear modal analysis will yield reasonably good results both for low and high force levels, though the modal parameters will be different in each case. Unlike cubic stiffness, nonlinear effects arising from friction damping cannot easily be detected by visual inspection alone.

- In mid-force ranges, the friction damper will become effective and the total damping values will be higher than those for inherent structural damping only (dotted line in Fig. 12). Therefore, damping errors are seen to be largest for those excitation forces (around $1000 \mathrm{~N}$ ) that yield maximum non-linear friction damping.

- As mentioned earlier, high force levels prevent relative motion and the damper locks. Therefore, the natural frequency tends to that of the linear system, shown in dotted line in Fig. 12(b).

\subsubsection{Non-linear modal analysis}

Detailed numerical studies of different underlying linear models indicated that the non-linear modal analysis procedure was sensitive to mode shape errors but 


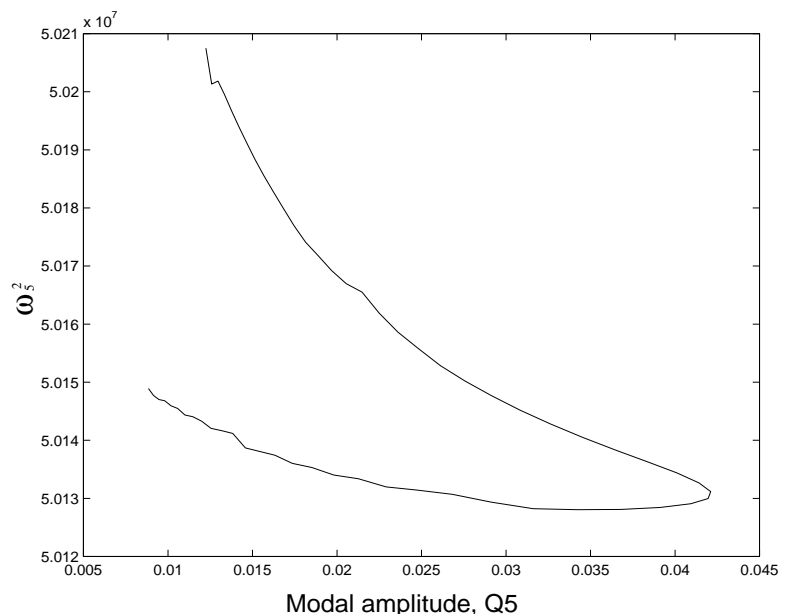

(a) Variation of natural frequency

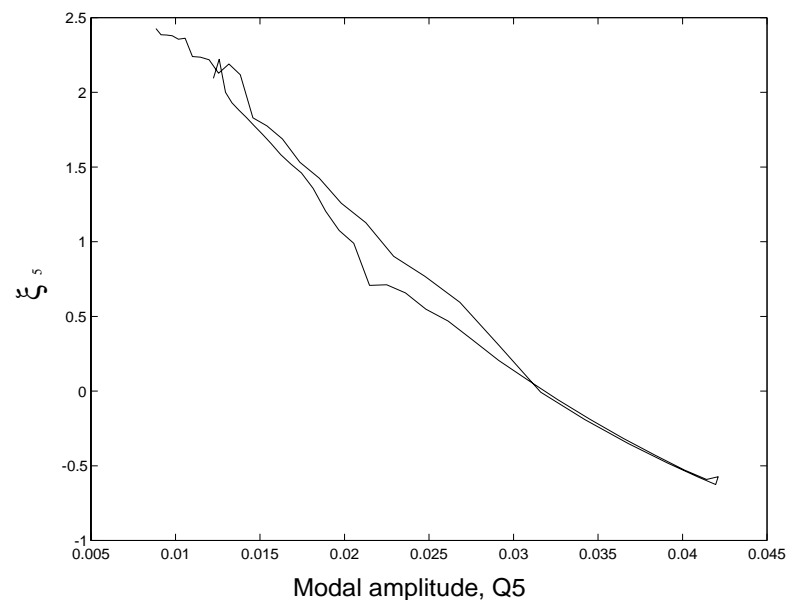

(b) Variation of damping ratio

Fig. 13. Effect of erroneous underlying linear linear model.

less so to other parameters. It was found that an erroneous linear mode shape could seriously undermine the accuracy of the results and such a case is shown in Fig. 13. While the variation of damping is consistent with the equivalent linearisation assumption, the same cannot be said about the variation of natural frequency which exhibits two distinct branches, leading to two distinct values for a single modal amplitude. It was found that this problem could be resolved if the underlying linear mode shape was more accurately estimated. Further numerical studies, not reported here, indicated that the coalescence of the two branches was a reliable indicator the suitability of the assumed underlying linear model. An underlying linear model was obtained on the basis of this criterion and a non-linear modal analysis was conducted for responses obtained for an excitation level of $500 \mathrm{~N}$. The variation of the extracted parameters is shown in Fig. 14.

The identified non-linear modal parameters were used to predict the response of the system at force levels of $500 \mathrm{~N}, 1000 \mathrm{~N}$ and $2000 \mathrm{~N}$. The findings were checked against HBM simulations and the results are plotted in Fig. 15. It is seen that there is good agreement between the two approaches, indicating success both for non-linear modal parameter identification and prediction of forced response from a single set of (simulated) non-linear measurements.

\section{Concluding Remarks}

- The experimental results indicate that the harmonic balance technique can accurately predict the re- sponse of systems with non-linear elements whose force-displacement characteristic is known. Compared to time-domain methods, such a frequencydomain approach is computationally very efficient.

- The experimental work demonstrates that is possible to obtain non-linear parameter variations from true measured response data. These variations can subsequently be used to predict response levels at other force levels.

- The technique is well suited for the study of large systems because of its modal-based representation. Furthermore, case studies indicate that nonlinear contributions are usually confined to a few neighbouring modes only, thus allowing the truncation of the "away" modes for computational efficiency.

- It is found that an approximate knowledge of the underlying linear system can be sufficient in most practical situations. Such a feature greatly improves the practical applicability of the non-linear modal analysis technique to engineering structures.

- The non-linear modal analysis technique has a number of limitations.

1. It will work best with weakly non-linear systems. However, its significance lies in the fact that its formulation is compatible with both FE methods and existing linear modal analysis techniques. Therefore, it is an incremental but significant improvement over current methods that ignore non-linear behaviour altogether. 


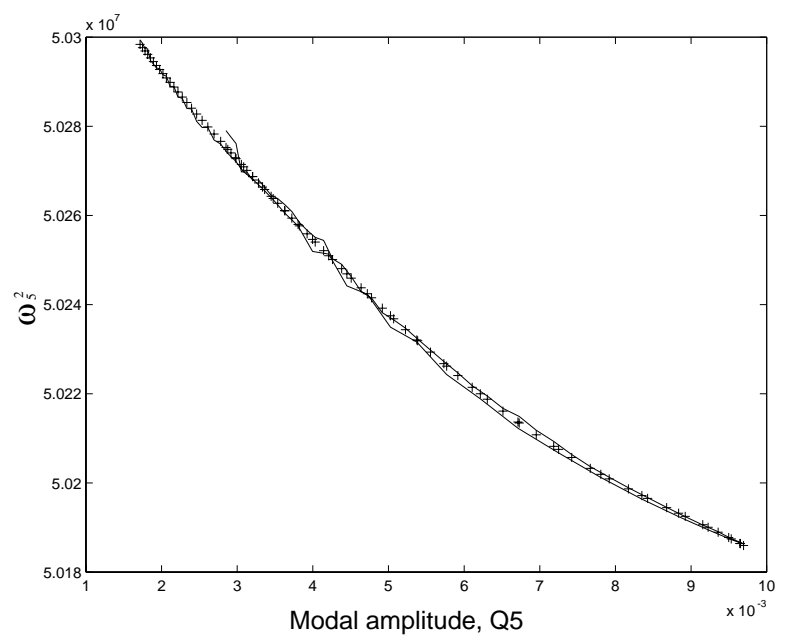

(a) Variation of natural frequency

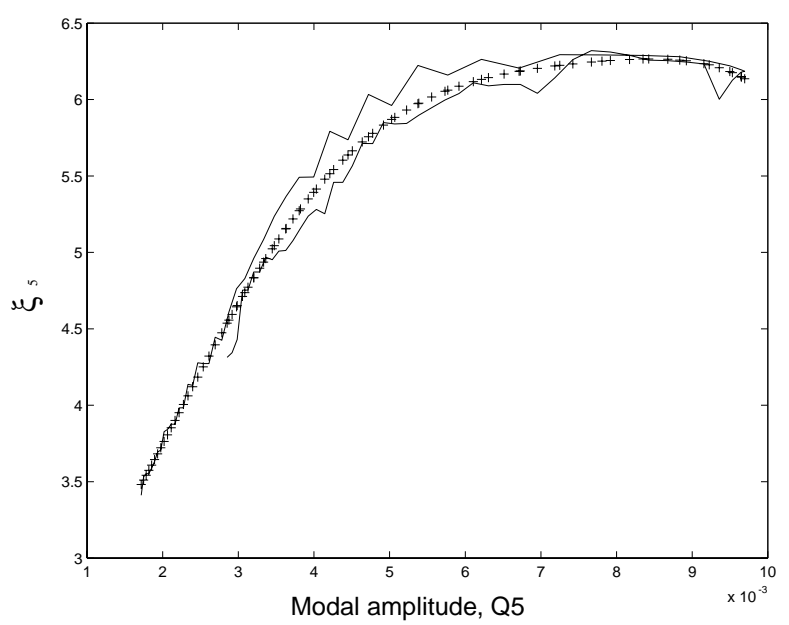

(b) Variation of damping ratio

Fig. 14. Variation of non-linear modal parameters: $* * * * *$ Extracted values, _- Curve-fitted values.

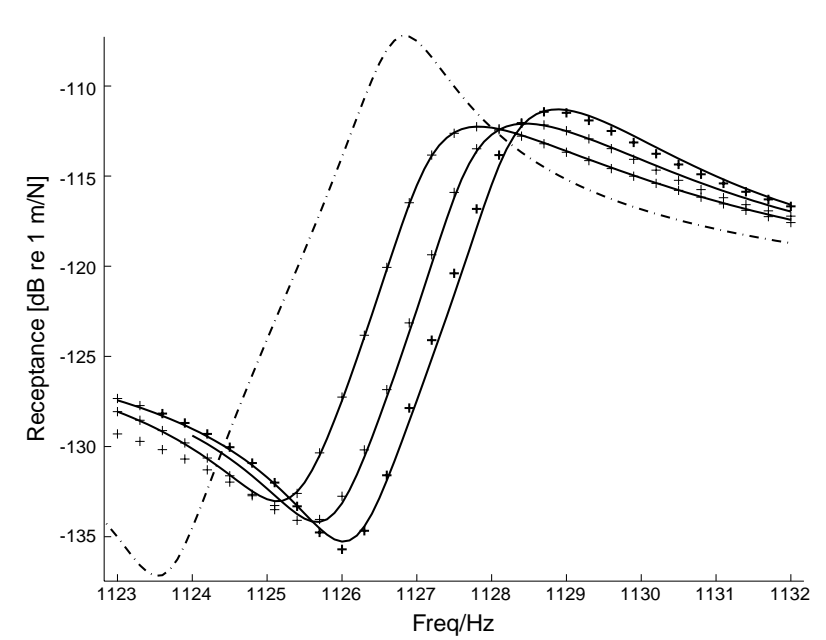

(a) Modulus plot

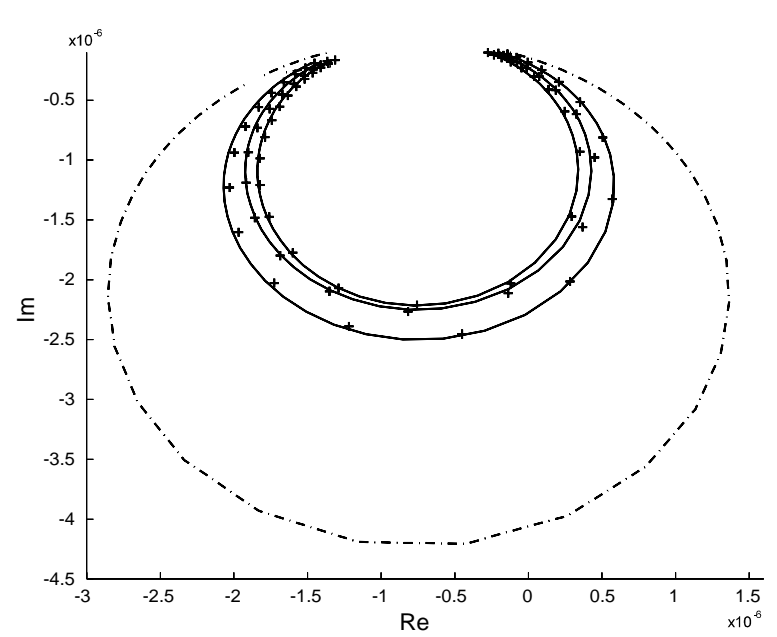

(b) Nyquist plot

Fig. 15. Predictions with assumed linear model: ... Linear response, _. HBM simulations, ***** Regeneration.

2. The modes need to be well separated in order to satisfy the non-linear normal mode assumptions.

3. The force levels at which the modal parameters are obtained and those for which the new response predictions are made must be within two orders of magnitude. This is especially true for non-linearities giving rise to complex modal parameter variations.

4. The analysis is based on the first harmonic of the response and hence it is not suitable for studying non-linearities that give rise to super or sub harmonics.

\section{References}

[1] C. Breard, A. Sayma, M. Vahdati and M. Imregun, A resonance tracking algorithm for the prediction of turbine forced response with friction dampers 45th ASME International Gas Turbine \& Aeroengine Technical Congress, May 2000, ASME paper 2000-GT-0372, 2000.

[2] M. Burdekin, N. Back and A. Cowley, Experimental study of normal and shear characteristics of machined surfaces in contact. Journal of Mechanical Engineering Science 180(20) (1978), 129-132. 
[3] J.V. Ferreira, Dynamic response analysis of structures with nonlinear components, $\mathrm{PhD}$ thesis, Imperial College, MED, Dynamics Section, 1998.

[4] L.E. Goodman and C.B. Brown, Energy dissipation in contact friction: constant normal and cyclic tangential loading, $J$. Applied Mechanics (1962), 17-22.

[5] C.H. Meng and J.H. Griffin, A comparison of transient and steady-state fe analyses of the forced response of a frictionally damped beam, Journal of Vibration, Stress \& Reliability in Design 107 (1985), 19-25.
[6] F. Pfeiffer and M. Hajek, Stick-slip motion of turbine blade damper, Philosophical Transactions of the Royal Society of London 338(1641) (1985), 503-517.

[7] K.Y. Sanliturk, M. Imregun and D.J. Ewins, Harmonic balance vibration analysis of turbine blades with friction dampers, Journal of Vibration \& Acoustics 118 (1996), 96-103.

[8] K.Y. Sanliturk, A.B. Stanbridge and D.J. Ewins, Friction damper optimization for turbine blades: experiment and theoretical predictions, IMechE Paper C500/019/96, 1996. 

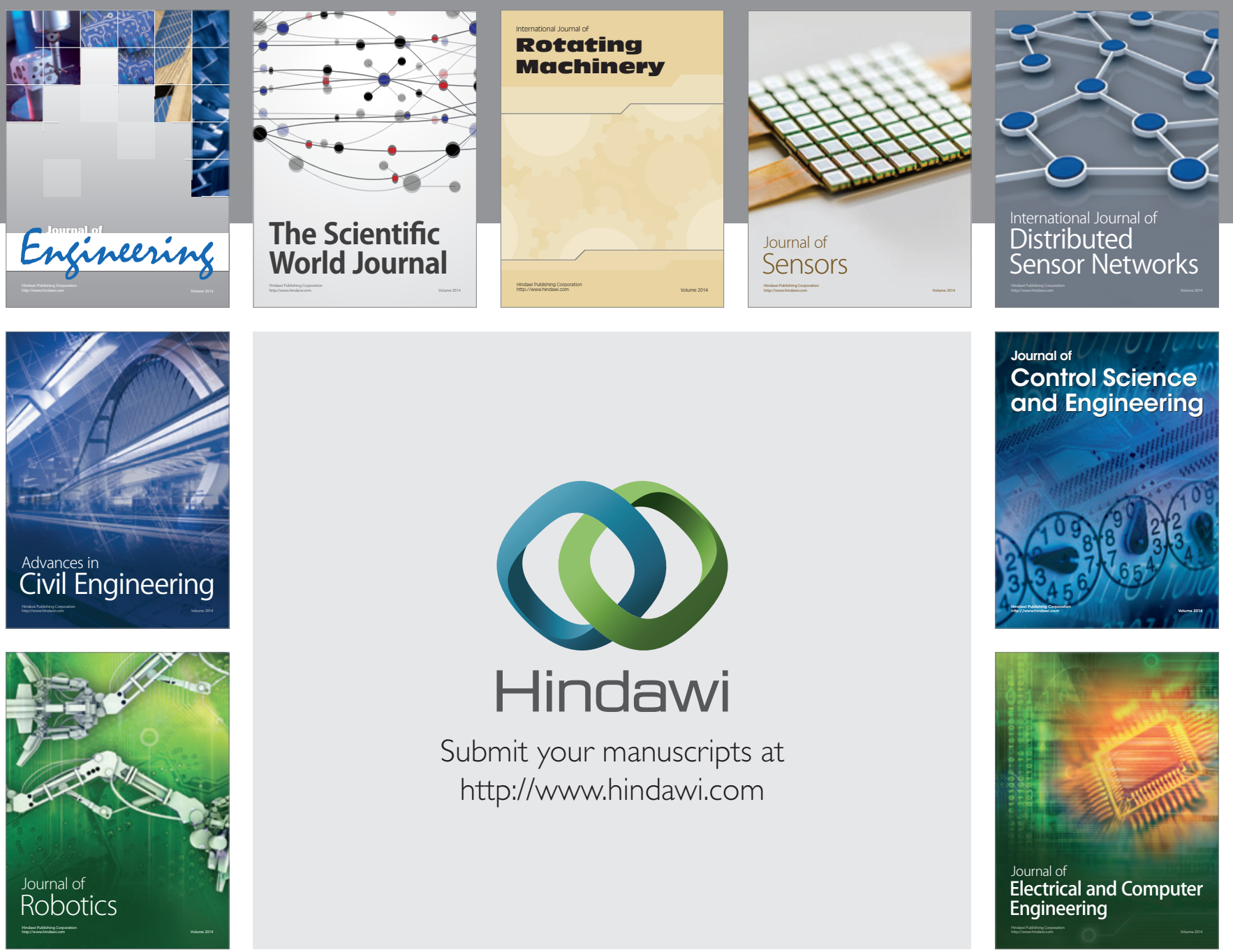

Submit your manuscripts at

http://www.hindawi.com
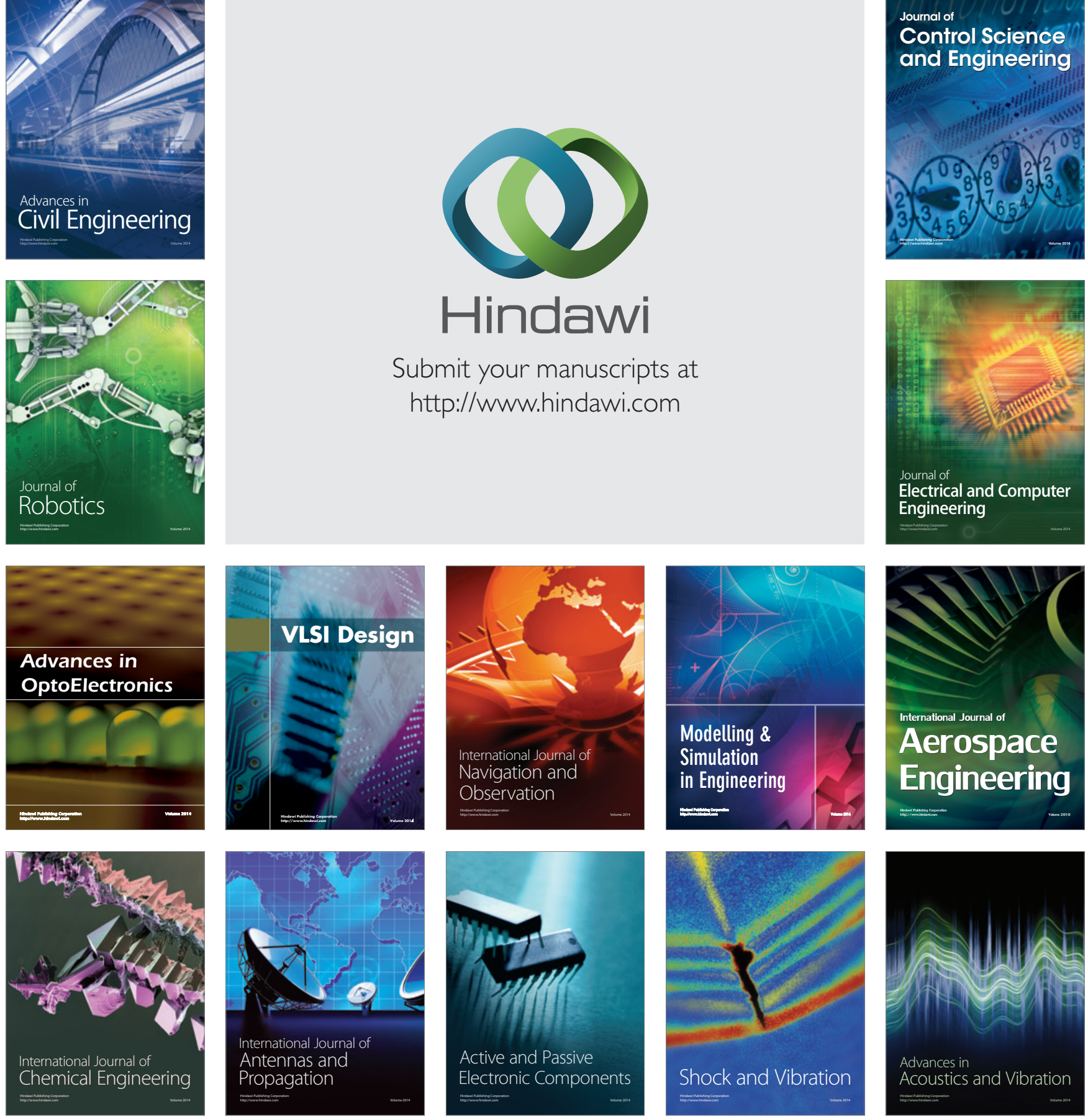